\title{
O QUESTIONÁRIO DE SUPORTE SOCIAL (SSQ): ESTUDOS DA ADAPTAÇÃO PARA O PORTUguÊs
}

\author{
Thelma S. Matsukura ${ }^{1}$ \\ Edna M. Marturano ${ }^{2}$ \\ Jorge Oishi
}

Matsukura TS, Marturano EM, Oishi J. O questionário de suporte social (SSQ): estudos da adaptação para o português. Rev Latino-am Enfermagem 2002 setembro-outubro; 10(5):675-81.

Com o objetivo de realizar a versão e estudos psicométricos do Questionário de Suporte Social de Sarason, aplicouse o instrumento em 113 mulheres-mães. Para a análise, utilizou-se a estatística Kappa, o Índice de Concordância Bruta e a Correlação de Spearman. A consistência interna do instrumento foi avaliada. Os resultados indicam uma fidedignidade testereteste aceitável e um alto nível de consistência interna. Observaram-se associações estatísticas entre os índices de suporte social e as variáveis trabalho, gravidez e renda. Apresentam-se informações sobre fontes de suporte social e extensão da rede as quais sugerem uma participação maior da família próxima como fornecedora de suporte.

\section{THE SARANSON'S SOCIAL SUPPORT QUESTIONNAIRE: STUDIES REGARDING ITS ADAPTATION TO PORTUGUESE}

With the aim of developing psychometrical studies using the Saranson's Social Support Questionnaire, an adapted version of the instrument was applied to 113 mothers. The Kappa Statistical Parameter, the Index of Gross Agreement and Spearman's Correlation were used to the analysis. The analysis of the internal consistency instrument showed a high level of it. In addition, the results indicated an acceptable test-retest reliability as well as statistical relationships between the social support indexes and variables related to work, pregnancy and income. Information on social support sources and the extension of the network suggest a greater participation of the family as the provider of the support.

\section{CUESTIONARIO DEL APOYO SOCIAL DE SARANSON: ESTUdIOS DE LA ADAPTACIÓN EN PORTUGUÉS}

Con el objetivo de realizar la versión y estudios psicométricos del Cuestionario del Apoyo Social de Saranson, se empleó el instrumento en 113 mujeres-madres. Para la análisis se utilizó la estatística Kappa, el Índice de Concordancia Bruta y la Correlación de Spearman. Se estimó la consistencia interna del instrumento. Los resultados muestran una fidelidad testretest acetable y un elevado nivel de consistencia interna. Se observaron asociaciones estadísticas entre los índices de apoyo social y las variables trabajo, gestación y renta. Se presentan informaciones sobre fuentes de soporte social y extensión de la redi, las cuales sugieren una mayor participación de la familia como proveedora del apoyo.

\footnotetext{
${ }^{1}$ Doutor pelo Programa de Pós-Graduação em Saúde Mental da Faculdade de Medicina de Ribeirão Preto da Universidade de São Paulo, docente da Universidade Federal de São Carlos, e-mail: thelma@power.ufscar.br; ${ }^{2}$ Professor Associado da Faculdade de Medicina de Ribeirão Preto da Universidsade de São Paulo; ${ }^{3}$ Professor Adjunto da Universidade Federal de São Carlos
} 


\section{INTRODUÇÃO}

Desde a década 70 até os anos 90 , tem sido observado um aumento nas pesquisas sobre suporte social ${ }^{(1-2)}$, denotando um interesse crescente pelo tema. Alguns autores ${ }^{(3)}$ refletem sobre o interesse no estudo do suporte social, em função de diversos fatores, dentre eles a importância de achados referentes à relação do suporte social, com indicadores de presença/ausência de diversas doenças e, no caso da presença da doença, a relação do suporte com as previsões de prognóstico e restabelecimento do indivíduo. De fato, vários estudos têm apontado a associação entre suporte social e níveis de saúde e/ou a presença de suporte social funcionando como agente "protetor" frente ao risco de doenças induzidas por estresse ${ }^{(4-6)}$.

Pesquisadores na área ${ }^{(7)}$ apresentam uma estrutura teórica dos aspectos presentes no domínio do suporte social, que podem estar relacionados à saúde ou ao estresse. Os três grandes aspectos propostos seriam: 1) Relacionamentos sociais (existência, quantidade, tipo); 2) Suporte social (tipo, fonte, quantidade ou qualidade) e; 3) Rede social (tamanho, densidade, reciprocidade, intensidade, dentre outros).

Em relação ao segundo grande aspecto, considera-se "fonte" a identificação do relacionamento estabelecido com o provedor de suporte, como, por exemplo, família e $\operatorname{amigos}^{(2)}$.

Ressalta-se ${ }^{(8)}$ que o suporte social deve ser considerado como um metaconstruto com três componentes conceituais distintos, ou seja, recursos de rede de suporte, comportamento suportivo e as avaliações subjetivas de suporte. Sob um enfoque ecológico, aborda como os recursos das redes sociais apresentam um "rendimento sustentável", ou seja, podem ser desenvolvidos, mantidos, cuidados e estimulados ou deteriorados, negligenciados e, até, destruídos, em função de variáveis como características dos indivíduos que compõem a rede, fatores ambientais ou culturais e interações entre essas variáveis.

Ainda são raras as publicações que tratam de suporte social no Brasil. Alguns autores ${ }^{(9-10)}$ têm focalizado aspectos teóricos do suporte social, bem como desenvolveram uma pesquisa sobre a associação entre suporte social e distúrbios psiquiátricos em mulheres infectadas pelo $\mathrm{HIV}^{(10)}$.

Considerando o interesse na investigação de relações entre suporte social e níveis de estresse em mulheres-mães, no contexto de uma pesquisa mais ampla à qual este estudo está vinculado, em que se investiga a avaliação da hipótese apresentada pelo modelo do efeito protetor do suporte social nas situações estressoras ${ }^{(5,11)}$, buscou-se um instrumento de medida que fornecesse dados de, pelo menos, três dimensões do metaconstruto, ou seja, a presença/existência de pessoas capazes de fornecer apoio em diferentes situações, a percepção/ identificação do apoio disponível e a avaliação sobre a situação de suporte. Como aponta a literatura ${ }^{(5)}$, foram encontradas consistentes evidências sobre o papel de suporte social, atuando como protetor nas situações estressoras quando as medidas de suporte estão relacionadas com a disponibilidade percebida de recursos interpessoais que repondem às necessidades presentes nos eventos estressantes.

Assim, selecionamos o Social Support Questionnaire $(\mathrm{SSQ})^{(12)}$, que fornece escores para o número de figuras de suporte percebido pelos respondentes e para a satisfação com o suporte social recebido. O SSQ é composto por 27 questões, sendo que cada questão solicita uma resposta em duas partes. Na primeira parte, deve ser indicado o número de fontes de suporte social percebido (SSQ-N), podendo o respondente listar até nove possibilidades (além da opção nenhum); na segunda parte, o respondente deve informar sobre sua satisfação com esse suporte (SSQ-S), fazendo uma opção em uma escala de 6 pontos (que varia de muito satisfeito a muito insatisfeito).

O Social Support Questionnaire foi submetido a vários estudos realizados pelos autores, para determinação de suas medidas psicométricas ${ }^{(1,12-13)}$. Na aplicação do instrumento a uma amostra de 602 estudantes universitários, o coeficiente alfa de Kronbach obtido foi de 0,97 para o SSQ-N e de 0,94 para o SSQ-S. A fidedignidade teste-reteste foi de 0,90 para o SSQ-N e de 0,83 para o SSQ-S, sugerindo que o SSQ é um instrumento estável e com alta consistência interna.

Os indicadores de validade foram baseados em comparações entre o SSQ e a MAACL - Multiple Affect Adjective Check List; e a LP-Lack of Protection Scale. Em um estudo utilizando a MAACL, com uma amostra de 277 estudantes, encontrou-se uma significante correlação negativa entre o SSQ-N e o SSQ-S e medidas de desconforto emocional da MAACL (ansiedade, depressão e hostilidade). As comparações com a escala LP também 
apresentaram correlação negativa (lembranças de ansiedade de separação na infância) com o SSQ-N e SSQ-S.

Assim, o objetivo do presente trabalho visa à tradução e à realização de alguns estudos psicométricos do instrumento de medida de suporte social.

\section{MÉTODO}

Participantes

Participaram desse estudo 125 mulheres (125 no estudo piloto, e 113 que responderam a, pelo menos, uma fase da aplicação do SSQ), mães de crianças com idade variando de 0 a 12 anos, moradoras em diferentes bairros da cidade de São Carlos, localizada no interior de São Paulo. Todas as participantes foram informadas sobre os objetivos da pesquisa e consentiram em participar.

As mães foram localizadas por meio de duas escolas públicas (42\%) e uma escola particular (26\%), assim como por indicação de outras mães (32\%).

A idade média das respondentes foi de 34 anos, sendo que $52 \%$ encontram-se na faixa etária entre 30 a 40 anos, e 32\%, entre 20 e 30 anos. A maioria das participantes cursou até o Primeiro Grau (46\%). Apenas $4 \%$ da amostra declarou a renda familiar até dois salários mínimos (correspondente a $\mathrm{R} \$ 302,00$ atuais), e a maioria declara renda familiar maior do que quatro salários mínimos (acima de $\mathrm{R} \$ 604,00$ atuais).

Em maioria, as participantes são casadas e vivem com seus companheiros $(75 \%)$, sendo que a média de número de filhos é dois (a maioria das mães têm dois ou apenas um filho). Apenas $3 \%$ das participantes encontravam-se grávidas. Com relação à ocupação, há um equilíbrio da amostra, aproximadamente $50 \%$ das mães não trabalha fora do lar, e $50 \%$ estão no mercado de trabalho.

\section{Procedimentos}

Tradução do instrumento de medida

A primeira autora, com o auxílio de um professor de inglês, realizou, na íntegra, a tradução do questionário de suporte social. Após a tradução, solicitou-se a sete juízes, pesquisadores com domínio no idioma inglês, que cotejassem a versão em português com o original em inglês, apontando inadequações e sugerindo modificações. Todas as questões cuja tradução foi avaliada como inadequada por pelo menos quatro juízes, foram modificadas; nos casos onde não houve consenso nas sugestões, optou-se por aquela apresentada pelo maior número de juízes.

O procedimento de retroversão foi realizado por meio da tradução da versão em português para a língua inglesa por um professor de inglês que já residiu nos dois países. Três juízes compararam a retroversão e a versão original em inglês, com o objetivo de avaliar possíveis discrepâncias de sentido dos itens presentes no questionário. As análises indicaram grande semelhança entre as duas versões que constituem indicação de validade de conteúdo das escalas ${ }^{(14)}$.

Estudo piloto

Realizou-se um estudo piloto, com três aplicações sucessivas do questionário em um total de 12 mulheres/ mães. A primeira aplicação foi grupal (4 mães), realizada com o objetivo de verificar as instruções e esclarecimentos necessários na situação da aplicação (leitura das perguntas pelo pesquisador, tempo necessário para a aplicação, etc.) e, para receber sugestões relativas à compreensão das perguntas, possíveis dificuldades para as respostas, etc.

Nas segunda e a terceira aplicações, o objetivo foi a avaliação da forma grupal ou individual para a apresentação do questionário. Tal procedimento indicou a adequação da aplicação individual. Considerou-se, também, que as questões deveriam ser apresentadas e preenchidas pelo aplicador.

\section{Aplicação do SSQ}

Inicialmente, foi estabelecida a estratégia para a aplicação dos questionários em uma amostra de 100 mães, visando ao estudo estatístico do SSQ, em dois momentos distintos (teste/reteste), respeitando-se um intervalo de 40 dias entre uma aplicação e outra, conforme o procedimento original adotado pelos autores do instrumento. Para a coleta de dados, contou-se com o auxílio de dois alunos do Curso de Graduação em Terapia Ocupacional da Universidade Federal de São Carlos, que foram orientados e treinados para tal fim. 
As mães foram avisadas do local, data e horário do encontro, recebendo um lembrete impresso por meio do seu filho (via professor/escola), ou diretamente do pesquisador (via carta ou entregue pessoalmente). Quando a mãe comparecia para o primeiro encontro individual, já era avisada da data, local e horário, e da importância do seu retorno no segundo encontro. Alguns dias antes do segundo encontro, a mãe era novamente contactada.

Na primeira fase de aplicação, responderam ao SSQ 113 mães; na segunda fase, 97 mães (86\% da amostra original) compareceram e responderam novamente ao SSQ. Assim, os indicadores de fidedignidade teste/reteste foram calculados sobre os dados dessas 97 mães.

\section{RESULTADOS E DISCUSSÃO}

A comparação entre as respostas do teste e do reteste para cada um dos 27 itens das escalas (SSQ-N e SSQ-S), foi feita utilizando a estatítica Kappa ${ }^{(15)}$. Neste trabalho, foi considerado o critério utilizado em estudos anteriores $^{(16)}$ onde encontramos três grupos de classificação: igual ou inferior a 0.29 - valores baixos e indicativos de um acordo pobre; de 0.30 a 0.49 - valores médios, indicativos de um acordo médio; e, acima de 0.50 - valores altos e indicativos de um bom acordo.

O coeficiente Kappa tem sido extensivamente usado para avaliar concordâncias em estudos de várias áreas, entretanto ele não é indicado nas situações em que há grande número de respostas nas extremidades da escala, como, por exemplo, quando numa escala de 5 pontos, em que as respostas são, na maioria, em torno de 1 (ou de 5), pode ocorrer o efeito da assimetria, isto é, se há tendência de a grande maioria das respostas ser 1 (ou 5). Isso provoca no Kappa um efeito perturbador para baixo, caso notado em alguns itens analisados nesse trabalho.

Assim, os valores de Kappa não devem ser considerados como indicadores absolutos da concordância entre o teste e o reteste, devendo ser levados em consideração também outros indicadores, conforme se apresenta nesse trabalho, como o Coeficiente de Correlação de Spearman ou o Coeficiente de Concordância Bruta, que é dado pela proporção de empates entre o teste e o reteste em relação ao número total de possibilidades.

Os dados relativos aos índices de estabilidade do SSQ. São apresentados na Tabela 1.

Tabela 1 - Índices de estabilidade do Questionário de Suporte Social (SSQ)

\begin{tabular}{|c|c|c|c|c|c|c|c|c|}
\hline \multicolumn{5}{|c|}{ SÉRIE N } & \multicolumn{4}{|c|}{ SÉRIE S } \\
\hline Item & $\begin{array}{c}\text { Concordância } \\
\text { Bruta\% }\end{array}$ & $\begin{array}{c}\text { Correlação } \\
\text { de } \\
\text { Spearman }\end{array}$ & Kappa & Classificação & $\begin{array}{c}\text { Concordância } \\
\text { Bruta } \%\end{array}$ & $\begin{array}{c}\text { Correlação } \\
\text { de } \\
\text { Spearman }\end{array}$ & Kappa & Classificação \\
\hline 1 & 53,1 & 0,742 & 0,42 & $\mathrm{M}$ & 63,3 & 0,572 & 0,34 & $\mathrm{M}$ \\
\hline 2 & 51,0 & 0,590 & 0,34 & M & 68,0 & 0,514 & 0,47 & $M$ \\
\hline 3 & 40,8 & 0,744 & 031 & M & 83,7 & 0,465 & 0,43 & $M$ \\
\hline 4 & 51,0 & 0,585 & 0,37 & M & 69,4 & 0,574 & 0,45 & M \\
\hline 5 & 46,9 & 0,548 & 0,27 & B & 70,4 & 0,649 & 0,44 & $M$ \\
\hline 6 & 49,0 & 0,701 & 0,30 & M & 76,5 & 0,709 & 0,58 & A \\
\hline 7 & 32,7 & 0,560 & 0,16 & B & 70,4 & 0,608 & 0,44 & M \\
\hline 8 & 62,2 & 0,670 & 0,51 & A & 71,4 & 0,680 & 0,49 & M \\
\hline 9 & 42,9 & 0,588 & 0,51 & A & 78,6 & 0,724 & 0,57 & A \\
\hline 10 & 51,0 & 0,612 & 0,34 & M & 66,3 & 0,530 & 0,41 & M \\
\hline 11 & 52,0 & 0,712 & 0,37 & M & 74,5 & 0,683 & 0,52 & A \\
\hline 12 & 40,8 & 0,485 & 0,31 & M & 75,5 & 0,547 & 0,44 & $M$ \\
\hline 13 & 40,8 & 0,546 & 0,25 & B & 75,5 & 0,654 & 0,57 & A \\
\hline 14 & 52,0 & 0,556 & 0,33 & M & 71,4 & 0,649 & 0,50 & A \\
\hline 15 & 43,9 & 0,465 & 0,27 & B & 77,6 & 0,648 & 0,54 & A \\
\hline 16 & 52,0 & 0,613 & 0,33 & $M$ & 74,5 & 0,568 & 0,50 & A \\
\hline 17 & 49,0 & 0,591 & 0,31 & M & 72,4 & 0,571 & 0,52 & A \\
\hline 18 & 50,0 & 0,594 & 0,36 & $M$ & 72,4 & 0,476 & 0,40 & $M$ \\
\hline 19 & 45,9 & 0,556 & 0,34 & $M$ & 68,4 & 0,623 & 0,43 & $M$ \\
\hline 20 & 53,1 & 0,567 & 0,38 & $M$ & 68,4 & 0,539 & 0,41 & $M$ \\
\hline 21 & 49,0 & 0,516 & 0,29 & B & 63,3 & 0,522 & 0,37 & $M$ \\
\hline 22 & 53,1 & 0,612 & 0,35 & $M$ & 73,5 & 0,679 & 0,54 & $A$ \\
\hline 23 & 50,0 & 0,600 & 0,36 & $M$ & 73,5 & 0,645 & 0,56 & A \\
\hline 24 & 35,7 & 0,608 & 0,25 & $B$ & 86,7 & 0,653 & 0,58 & $A$ \\
\hline 25 & 40,8 & 0,437 & 0,24 & $B$ & 71,4 & 0,669 & 0,55 & $A$ \\
\hline 26 & 54,1 & 0,687 & 0,37 & $M$ & 77,6 & 0,718 & 0,58 & $A$ \\
\hline 27 & 53,1 & 0,572 & 0,36 & $\mathrm{M}$ & 69,4 & 0,629 & 0,50 & A \\
\hline
\end{tabular}


As médias de classificação Kappa obtidas para os escores SSQ-N e SSQ-S são: para a escala N, média de 0,33 e desvio padrão de 0,074 ; para a escala $S$, média de 0,49 e desvio padrão de 0,070 (ambas com classificação Kappa: médio).

Os Coeficientes Alfa de Kronbach, que medem a consistência interna em cada fase (teste e reteste) obtidos, foram: para a Escala N (SSQ-N), 0,9384 no teste e de 0,9610 no reteste. Para a Escala S (SSQ-S), 0,9438 no teste e 0,9626 no reteste.

Como se depreende dos dados da Tabela 1, na escala N (SSQ-N), 7,4\% dos itens obtiveram classificação alta, $66,7 \%$ classificação média e $25,9 \%$ classificação baixa. Dos 27 itens da escala S (SSQ-S), 51,8\% obtiveram classificação alta, $48,2 \%$, classificação média, e nenhum item foi classificado como baixo.

Deve ser salientado que, na escala N, havia 10 categorias de respostas, enquanto, na escala $\mathrm{S}$, apenas seis. No caso de 10 categorias, pelo seu refinamento, ou seja, pela maior dificuldade de localização precisa das respostas em relação à escala de seis categorias, não é recomendável a comparação entre os indicadores das duas escalas. Os valores mais baixos na escala de 10 categorias devem-se, principalmente, a esse fato, ou seja, é muito mais difícil haver concordância entre as respostas do teste e do reteste pela dificuldade dos respondentes em localizar precisamente as mesmas respostas nas duas situações.

Assim, pode-se considerar que o Questionário de Suporte Social apresentou uma fidedignidade teste-reteste satisfatória, levando-se em conta os três índices utilizados, bem como um alto nível de consistência interna.

Apresentaremos a seguir alguns resultados encontrados na amostra utilizada neste estudo, relacionando os níveis de suporte social ( $\mathrm{Ne} \mathrm{S}$ ) presentes com variáveis sociodemográficas. Para tanto, utilizou-se o escore médio de cada escala (série $\mathrm{N}$ e série $\mathrm{S}$ ), o teste não paramétrico de Kruskal-Wallis foi aplicado.

Foram incluídas nesta análise nove variáveis: escolaridade, estado civil, presença de companheiro, trabalho, período de trabalho, número de filhos, gravidez, moradia e renda familiar.

Os resultados indicaram que as mães que não trabalham fora apresentam maiores índices de suporte na escala N. As mães grávidas apresentaram menores índices de suporte na escala $\mathrm{N}$ e menor índice de satisfação com o suporte social recebido do que as mães que não estavam grávidas. As mães que declararam renda maior que $\mathrm{R} \$ 604,00$ apresentam maior suporte na escala $\mathrm{N}$ do que as mães que declararam menor renda (dentre as menores rendas não há diferenças na escala $\mathrm{N}$ ).

Os resultados permitem reflexões importantes e que podem contribuir para a compreensão do processo de suporte social. Ao buscarmos compreender por que as mulheres-mães que trabalham fora de casa, ou seja, que estão inseridas no mercado de trabalho, apresentam uma rede de suporte menor do que as mães que realizam o trabalho em casa, podemos encontrar na abordagem ecológica do suporte social algumas explicações.

Como tem sido relatado na literatura ${ }^{(17-18)}$, a maioria das mulheres inseridas no mercado de trabalho vivenciam a realidade da dupla jornada de trabalho, que envolve realizar as atividades inerentes ao seu emprego, bem como as tantas outras relacionadas à organização da rotina da família, cuidados com os filhos, etc. (atividades essas ainda marcadamente femininas). Uma das conclusões da pesquisa ${ }^{(17)}$ realizada com trabalhadoras brasileiras $\mathrm{e}$ italianas é que, para as trabalhadoras de ambos os países, a dupla jornada de trabalho das mulheres torna precárias as condições de vida, de realização do prazer e da sexualidade.

Como observado( ${ }^{(8)}$, as redes sociais são sustentadas e necessitam ser desenvolvidas e mantidas. Ë possível que as mães inseridas no mercado de trabalho tenham condições menos favoráveis tanto de desenvolvimento como de manutenção de sua rede social. Provavelmente essas mulheres têm menos tempo para se dedicar às relações pessoais (vizinhos, amigos, etc) e sociais (participação em atividades sociais, grupos, associações, etc).

Sob um enfoque semelhante, é possível levantar elementos para a compreensão da associação demonstrada entre renda familiar e tamanho da rede. Melhores condições econômicas podem interferir no processo de desenvolvimento e manutenção de redes de apoio, na medida em que o fator econômico pode estar relacionado com maior disponibilidade de tempo para os relacionamentos, maior escolaridade e melhores empregos, dentre outros.

Com relação às mulheres grávidas da amostra, foi possível identificar que elas estão na faixa de 21 a 26 anos, não trabalham fora, têm apenas um filho e declaram renda familiar na faixa de 2 a 4 salários mínimos. As mulheres grávidas da amostra apresentaram menor 
satisfação com o suporte social recebido e menor rede social do que as mães não grávidas.

É possível que uma variedade de fatores associados estejam presentes na determinação desses resultados, como, por exemplo, os diferentes graus de afetos e ansiedades presentes durante a gestação e a realidade socioeconômica dessas mães, interferindo no processo de desenvolvimento, manutenção da rede social e avaliação sobre o suporte social recebido. Como apontado na literatura social $^{(17,19)}$, o significado e as implicações da maternidade na vida da mulher estão correlacionados com as condições da realidade social e econômica em que vive e não apenas no desempenho da natural função de procriar.

De forma semelhante ao estudo das associações entre suporte social percebido e variáveis sociodemográficas, foi feita uma exploração dos resultados no que diz respeito ao tamanho da rede e às fontes de suporte, tendo por base os dados da amostra de 113 mães.

Foi possível observar que, em média, as mães citam uma rede constituída de cinco a seis pessoas (o total permitido pelo instrumento é nove), já a freqüência de opção pela alternativa "ninguém" é pequena, aparecendo, em média, em quatro dos 27 itens do SSQ.

Foi possível, também, identificar nessa amostra as fontes de suporte e a freqüência com que são citadas ao longo das 27 questões que compõem o instrumento. Esses dados podem ser obtidos desde que o respondente informe, em cada questão, o tipo de relacionamento que mantém com a(s) pessoa(s) citada(s). Observou-se que os maridos são os mais freqüentemente citados como fonte de suporte (correspondendo a $22 \%$ do total de 6180 citações feitas pelas 113 respondentes), seguidos por amigas(os) (19\%) e filhos (18\%). As demais fontes são distribuídas entre pessoas da família (tios, sogros, avós, etc), profissionais especializados (terapeuta, advogado, diretor da escola, etc) e conselheiros religiosos.

\section{REFERÊNCIAS BIBLIOGRÁFICAS}

1. Vaux A. Social Support: theory, research and intervention. New York: Praeger; 1988.

2. Winemiller DR, Mitchell E, Sutliff J, Cline DJ. Measurement strategies in social support: a descriptive review of the literature. Clin Psychol 1993; 49(5):638-48.

3. Cohen S, Syme SL. Social Support and Health. Londres: Academic Press; 1985.

4. Bloom JR. The relationship of social support and health. Soc Sci Med 1990; 30(5):635-7.

5. Cohen S, Wills TA. Stress, Social Support, and the Buffering Hypothesis. Psychol Bull 1985; 98(2):310-57.
Observam-se os relacionamentos familiares como uma importante fonte de suporte dessa amostra, que se evidencia ainda mais agrupando marido, filhos e parentes. Tal resultado poderia vir a caracterizar uma tendência mais atual de contatos sociais/familiares.

\section{CONCLUSÕES}

Os resultados dos estudos de adaptação e fidedignidade do SSQ realizados indicam uma fidedignidade teste-reteste analisada como aceitável, bem como um alto nível de consistência interna do instrumento. Acredita-se que o presente estudo possa contribuir para as pesquisas na área de suporte social no Brasil.

Os dados relativos à amostra utilizada para esse estudo indicam a associação entre o trabalho das mães fora do âmbito familiar e a gravidez com um tamanho de rede social menor do que os das mães que não estão no mercado de trabalho e não estão grávidas. As mães que declararam renda maior que quatro salários possuem uma rede social maior que as mães com menor renda. Com relação à satisfação com o suporte social recebido, as mães grávidas apresentam menor satisfação.

As características da rede social da amostra revelam que as maiores fontes de suporte social das mulheres/mães são o cônjuge e os filhos, seguidos dos parentes próximos. Tais dados podem indicar uma tendência nos relacionamentos atuais, onde a situação e as relações familiares passam a ser importante fonte de apoio.

Aponta-se para a importância e a necessidade de desenvolvimento de estudos no âmbito do suporte social, na medida em que o mesmo pode contribuir para a compreensão dos processos de saúde/doença, de qualidade de vida e, conseqüentemente, para as propostas de intervenções junto à população.

6. Pearlin LI. The sociological study of stress. Health Social Behavior 1989; 30:241-56.

7. House JS, Kahn RL. Measures and concepts of social support. In: Cohen S, Syme SL, organizer. Social Support and Health. Londres: Academic Press; 1985. p.83-105.

8. Vaux A. An ecological approach to understanding and facilitating social support. J Soc Pers Relat 1990; 7:507-18. 9. Lovisi GM, Milani I, Caetano G, Abelha L, Morgado AF. Suporte social e distúrbios psiquiátricos: em que base se alicerça a associação? Informação Psiquiátrica 1996; 15(2):65-8.

10. Lovisi GM, Morgado AF. Suporte social e distúrbios psiquiátricos em mulheres infectadas pelo HIV. Bras Psiquiatr 1996; 45(10):593-9. 
11. Castro R, Campero L, Hernández B. La investigación sobre apoyo social en salud: situación actual y nuevos desafíos. Rev Saúde Pública México 1997; 31(4):425-35.

12. Sarason IG, Levine HM, Basham RB, Saranson BR. Assessing social support: The Social Support Questionnaire. J Personal Social Psychol 1983; 44(1):127-39.

13. Heitzman CA, Kaplan RM. Assessment of Methods for Measuring Social Support. Health Psychol 1988; 7(1):75-109. 14. Silva MED, Novo R, Peralta E. Estudo exploratório para a adaptação portuguesa das escalas de desenvolvimento da personalidade de C. Ryff. In: Gonçalves M, organizador. Avaliação Psicológica: formas e contextos. Braga: APPORT; 1997. p. 453-60.

15. Cohen J. A coefficient of agreement for nominal scale. Educ Psychol Measurement 1960; 20:37-46.

16. Graminha SSV. A escala comportamental infantil de Rutter A2: estudos de adaptação e fidedignidade. Estudos Psicol 1994; 11(3):34-42.

17. Oliveira EM. A mulher, a sexualidade e o trabalho. São Paulo (SP): Hucitec/CUT; 1999.

18. Saffioti HIB, Muñoz-Vargas M. Mulher brasileira é assim. Rio de Janeiro (RJ): Rosa dos Tempos; 1994.

19. Roy L. O modo de ser mulher trabalhadora na reestruturação produtiva. Campinas (SP): Alínea; 1999. 